Catégorisation et classification, enjeux de pouvoir

\title{
En un combat douteux
}

Concurrence pour la conformation sexuée des Français d'ascendance migrante et coloniale

Nacira Guénif-Souilamas

\section{(2) OpenEdition}

12 Journals

Édition électronique

URL : https://journals.openedition.org/remi/2493

DOI : $10.4000 /$ remi.2493

ISSN : $1777-5418$

Éditeur

Université de Poitiers

Édition imprimée

Date de publication : 1 juin 2005

Pagination : 91-109

ISBN : 2-911627-40-7

ISSN : 0765-0752

Référence électronique

Nacira Guénif-Souilamas, «En un combat douteux », Revue européenne des migrations internationales [En ligne], vol. 21 - n² | 2005, mis en ligne le 01 octobre 2008, consulté le 15 avril 2022. URL : http:// journals.openedition.org/remi/2493; DOI : https://doi.org/10.4000/remi.2493

Ce document a été généré automatiquement le 15 avril 2022

(c) Université de Poitiers 


\title{
En un combat douteux
}

\author{
Concurrence pour la conformation sexuée des Français d'ascendance \\ migrante et coloniale
}

\author{
Nacira Guénif-Souilamas
}

1 En France, le vingtième siècle a été dominé par une conception de la cohésion sociale fondée sur l'intégration entendue comme entreprise de conformation. Elle fut successivement mais aussi simultanément assimilationniste et universaliste égalitariste, du tournant du dix-neuvième siècle au terme des "trente glorieuses ", inaugurant ce qui apparaît de plus en plus comme une période postcoloniale, postnationale et postindustrielle. Les multiples formes de socialisation qui ont alors prévalu avaient pour objet l'intériorisation d'un ensemble de normes et de codes mettant en adéquation les statuts individuels et collectifs avec les rôles sociaux et politiques de ces mêmes individus et collectifs. La forte "coalescence ", comme l'énonce la sociologie classique, entre statut et rôle et donc la conformation des individus à un ordre social et politique légitimé par la rhétorique du progrès a constitué une réalité et un horizon tout au long de la première modernité - cette période sera entendue ici comme un processus de progrès partagé et généralisé promettant des rapports sociaux amendables et perfectibles. Cette conception pratique et politique du monde se tournait vers cet horizon progressiste et égalitaire, confortée par l'analyse des dominations structurelles démontrant que les hiérarchies sociales étaient immuables tant que le progrès était confisqué au bénéfice d'une classe. Ce premier moment de la modernité atteste d'une conception holiste de la société où les identités sont massives et socialement déterminées; leur épaisseur sociale est telle que les sociologues se dispenseront d'en examiner les modes de production par des outils théoriques ou des objets de recherche. Lorsqu'à la fin du $\mathrm{XX}^{\mathrm{e}}$ siècle se déploient de vastes mutations, la crise devient un objet d'analyse nécessaire pour en comprendre les manifestations ; apparaissent alors les questions croisées de la crise du système industriel et la fin du mouvement ouvrier (Rancière, 1981; Touraine, 1973), la crise des identités (Dubar, 2002) ou encore la crise du modèle républicain d'intégration (Lapeyronnie, 1993). La mutation paradigmatique de la modernité met à jour son caractère incertain et consubstantiel du risque (Beck, 1986 ; Giddens, 1989 ; Latour, 1991). Cette mise à jour inédite souligne du même coup la vacuité autant que le trop-plein de l'expérience et les 
incertitudes éprouvées par des individus exposés au risque identitaire d'être soi (Ehrenberg, 1998; Kaufman, 2004). La norme d'individuation prenant graduellement une place centrale, il est devenu de plus en plus difficile de décliner l'invitation à l'individuation et les risques qui lui sont inhérents. Le passage d'un monde massif tenu par des récits collectifs à des mondes défaits et refaits par la multiplicité des sphères de vie et de différenciation balise l'espace mouvant dans lequel se jouent ces aventures. Qu'elles soient sexuées ou post-genre, nationales ou ethniques, politiques ou culturelles, les identités en gestation occupent et préoccupent, affectent et transforment dès lors tant les personnes que les systèmes.

2 Les Français d'ascendance migrante et coloniale incarnent une figure idéaltypique du passage hésitant d'une modernité à l'autre et en révèlent un des aspects les plus problématiques : la difficulté à être soi alors même que l'on est réduit à tenir le rôle, souvent à haut risque, de l'Autre. La question articulée et cumulative des mutations économiques et sociales, des revendications identitaires et du découplage entre nationalité et citoyenneté se cristallise en eux et occupe désormais une place propre dans l'analyse sociologique. Car loin d'être réductibles à une origine et/ou une culture indépassables, ces Français d'ascendance migrante et coloniale expriment la quintessence de processus ambivalents donc inconfortables de production de soi parce qu'ils subissent un régime de rareté matérielle et symbolique accentué par une modernité incertaine. Pour en comprendre le cadre et les contours, il importe dès lors de retracer les lignes de partage et de partition de parcours soumis à une injonction paradoxale illustrant les registres désajustés, familiaux, sociaux, structurels, dans lesquels se construisent et se vivent ces nouveaux Français. En vue d'une analyse des rapports sociaux sexués entre descendants de migrants et entre ceux-ci et leurs contemporains et des stéréotypes qui en surgissent, je redéploierai ici la problématique de la double assignation à la différence comme injonction paradoxale, appliquée aux «beurettes » dans un précédent travail (Guénif-Souilamas, 2003). Pour des raisons historiques et politiques, je renforce ce cadre analytique de la double injonction paradoxale, dont les manifestations jalonnent l'histoire longue des migrations vers le monde occidental, en l'enchâssant dans l'ensemble plus vaste de la question de la décolonisation à l'ère de la mondialité. En effet, pour la première fois les descendants de migrants devenus Français ont des ancêtres qui furent colonisés et/ou esclaves. Même si les deux expériences ne sont pas réductibles l'une à l'autre, même s'il existe en France aujourd'hui des immigrants qui ne sont pas venus d'anciennes colonies, la question coloniale et postcoloniale occupe une place centrale dans l'élucidation de l'injonction à la conformité, dans les processus de conformation et dans les conflits de normes qu'ils induisent. La raison principale de cette centralité n'est pas tant que des personnes soient marquées par le stigmate de l'indigénat ou de l'esclavage ou héritières d'un récit collectif lié à ce moment d'histoire commune et des mémoires conflictuelles qu'ils façonnent. Mais elle tient au fait que l'impensé colonial prend en otage les processus identificatoires de ces personnes en continuant de produire des effets pratiques, routinisés dans l'administration de la société, mais aussi symboliques, insidieusement présents dans la rhétorique républicaine. En raison de ce piège mémoriel, retour de balancier d'une amnésie prolongée, mais aussi et surtout réel, révélé par l'inflation des actes discriminatoires et des discours racistes, la société française est structurellement prise à partie par ce processus inachevé de la décolonisation et s'avère incapable d'en assumer les conséquences postcoloniales. Dès lors cette question n'épargne personne. Elle ne touche pas une fraction de la population 
en raison de son origine, ou des factions qui seraient contraintes de s'affronter du fait d'un passé indigeste, mais bien l'ensemble de la société. L'approche postcoloniale ne procède pas selon une logique essentialiste désignant les Arabes, les Noirs et les «Indochinois » à une condition propre, un destin commun indépassable. À l'inverse elle élabore une causalité constructiviste qui décèle, bien au-delà de ses objets "naturels", les signes de l'effectivité coloniale dans des formes de domination et d'inégalité légitimées de façon subliminale et inconsciente. En cela ce processus désigne les résidus d'un ordre révolu qui irrigue les questions identitaires et la rhétorique de l'égalité et affecte au premier chef le rapport à une altérité incarnée, que ce soit les anciens et nouveaux immigrants ou les nouveaux Français (Stora, 1999; Vergès, 2004 ; Bancel, Blanchard et Lemaire, 2005).

\section{Modernité de la migration, migration post-patriarcale}

3 En premier lieu, l'examen des rapports entre genres et générations des descendants de migrants et de leur société impose de prendre ses distances avec l'idée que les entrepreneurs de morale (Becker, 1985 : 171-187) immigrants n'appartiendraient pas au même monde que les entrepreneurs de morale se constituant en France. Qu'il s'agisse d'un côté des parents ou de membres de fratries et de parentèles plus ou moins étendues auxquelles s'agrègent des sages autoproclamés et des alliés plus ou moins fiables et de l'autre d'acteurs institutionnels ou auto-institués, d'autorités politiques, intellectuelles ou médiatiques, fondant leur légitimité sur le témoignage à la première personne ou sur une économie compassionnelle aux contours de plus en plus extensibles, l'entreprise de morale est aujourd'hui florissante et puise sa vigueur et sa vitalité dans la lutte concurrentielle à laquelle tous s'adonnent sans toujours disposer des mêmes armes. Les uns et les autres participent d'une modernité multiple et multiforme jusque dans les contradictions qu'elle nourrit (Göle, 2005). Processus tout autant inscrit dans la contemporanéité que l'est l'appel à l'intégration, la migration et ses vicissitudes contribuent à rendre visibles les entrepreneurs de morale qui agissent en son nom, et simultanément à invisibiliser ceux qui se prévalent du point de vue majoritaire, les légitimant dans le même mouvement aux yeux de leurs détracteurs (Guénif-Souilamas, 2002). Les uns comme les autres interagissent avec les générations qui se succèdent et s'affrontent à cette gageure, même si par contraste le conflit de génération semble souvent n'exister qu'entre les migrants et leurs descendants. Dès lors, le " poids de la tradition ", auquel se réfèrent des acteurs de tous ordres mais aussi des écrits entre autres sociologiques, n'est rien d'autre que la mise en forme de la concurrence déloyale entre les dépositaires d'injonctions dont le contenu diffère mais qui partagent un modus operandi et un objectif de conformation : l'imposition de normes présentées comme intangibles. Qu'il s'agisse de fidélité coutumière ou d'intégration par émancipation, les deux assignations tracent le même sillon tout en semblant aller dans des directions divergentes: d'un côté un retour collectif et imposé vers une tradition, fiction intemporelle, de l'autre l'accès individuel au progrès, signe de modernité. La double assignation est un analyseur qui permet de prendre ses distances avec le sens commun et de lire les effets, les conflits et les résonances individuelles et collectives de ces pressions normatives concurrentielles exercées dans l'environnement familial et institutionnel et entretenues par un climat médiatique accentuant les difficultés jalonnant la vie des migrants et en tenant ceux-ci pour responsables. Contredisant ces stéréotypes faciles, les tensions entre les parents migrants et leurs enfants donnent à 
voir l'épuisement de la tradition du fait même de la migration et son inéluctabilité dans le moment post-migratoire. Ce n'est pas la persistance de la tradition qui creuse le fossé entre la société « d'accueil » et les familles migrantes mais bien la position illégitime et le régime de contrainte imposés à ces familles lorsqu'elles recomposent un rapport à la modernité, moteur de la migration elle-même, objet d'une perpétuelle réévaluation et de constants réajustements. Plutôt que d'immobilisme, de réaction ou de régression ne pouvant susciter que réprobation et stigmatisation, il s'agit d'apprécier une dynamique de détraditionnalisation déployée en régime de modernité tardive, dont l'issue est donc incertaine.

4 Ainsi, les résistances à cette dynamique sont d'autant plus dénoncées qu'elles revêtent un caractère spectaculaire. Elles sont d'autant plus spectaculaires qu'est puissante cette dynamique de sortie de la tradition et, contrastée, ambivalente et différenciée l'adhésion des migrants et de leurs descendants à celle-ci. Les erreurs d'interprétation de ces parcours et leur renvoi à une anti-modernité ou à une extra-modernité sont autant le fait d'un outillage conceptuel et méthodologique inadapté que d'une mauvaise foi idéologique indexée à une doctrine intégrationniste trop peu problématisée. En effet, la constitution du champ de recherche sur les migrations tout comme la rupture épistémologique introduite par l'anthropologie postcoloniale a fourni l'opportunité de décentrer le regard sur les migrants comme toute population allochtone et ainsi de réévaluer les approches culturalistes, souvent mâtinées d'évolutionnisme, tout comme les approches fonctionnalistes normatives, aucune ne permettant de faire une sociologie rigoureuse et une anthropologie imaginative des processus migratoires. L'écart à la norme, la mesure des déviances constitués comme outils imparables ont trop longtemps contribué à entretenir des angles morts dans la connaissance des faits migratoires et à accréditer certaines assertions relayées par les institutions et les médias. Parce qu'elle entretient une veine misérabiliste et stigmatisante, ethnocentrée, cette perception tend à disparaître du champ disciplinaire mais certes pas du champ politique et idéologique. Nombre d'acteurs institutionnels et politiques sont prêts à acheter l'interprétation culturaliste des "retards d'adaptation» des migrants et les mots d'ordre normatifs mesurant le «déficit d'intégration" de leurs enfants sans prendre la mesure de l'anachronisme d'un tel diagnostic et ses effets désastreux sur ce qu'ils prétendent défendre : la cohésion sociale. Sauf à considérer indûment les effets pratiques d'une prophétie auto-réalisatrice, il est donc moins question d'opposer tradition et modernité en un hypothétique choc de cultures dont il conviendrait de mesurer la réalité et les conséquences, que de voir la double assignation comme les deux faces d'une même volonté de conformation qui se heurte aux désirs d'individuation et donc de différenciation de ses destinataires. Une volonté de conformation qui, en définitive, s'affronte de manière problématique aux transformations sociétales imprimées par la modernité contemporaine.

\section{Sens et sexuation d'une entreprise de conformation}

5 Si l'on considère le sens qu'attribuent les entrepreneurs de morale cités ci-dessus à l'injonction apparemment contradictoire à l'intégration-émancipation ou à la fidélité coutumière, il est frappant de constater que l'une comme l'autre se répondent, se complètent en ce qu'elles s'appuient sur le rejet mutuel de règles et de comportements jugés contradictoires ou antinomiques. Il ne serait pas possible dans l'esprit de leurs 
promoteurs de concilier et moins encore de réconcilier des horizons si diamétralement opposés, des impératifs procédant de fondements philosophiques aussi étrangers l'un à l'autre. Et pourtant...

\section{Sens communs}

6 Si l'intégration s'adresse à des individus achevés ou en devenir c'est pour leur accorder la plénitude de leurs droits selon une perspective égalitariste abstraite, qui voilerait, pour mieux les ignorer, les origines et les particularités dont ils sont dépositaires. Il s'agirait de les remplir d'autonomie et de liberté en les vidant d'appartenances et de dépendances jugées comme autant d'entraves à leur émancipation. À cette fin, même les traces résiduelles, les richesses attachées à leur histoire, les secrets inviolés, les souvenirs et les usages soigneusement sélectionnés obéissent à cette entreprise de conformation : témoignage ou vestige du passé assumé parce que définitivement révolu et atténué dans ses effets (Lepoutre, 2005).

7 Symétriquement, la fidélité coutumière s'attache précisément à la persistance et la pérennisation de signes et d'actes qui indiquent que le passé ne s'est pas dissous en immigration, que des usages re-signifiés au sein et au cours de la dynamique migratoire ne sont pas condamnés à la disparition en raison de leur inadaptation au présent. Conçue comme anachronique, l'injonction à la fidélité coutumière est au contraire inventée en temps réel et repose sur l'illusion que le temps n'a pas d'effet sur ceux qui y sont attachés. Or c'est en raison même du temps écoulé, déployé et des marques qu'il appose sur des individus déplacés dans des espaces nouveaux et mouvants et tenus de reconsidérer les rapports qu'ils entretiennent les uns avec les autres que la fidélité s'impose comme une observance nécessaire et intangible. Observance d'autant plus attendue des immigrants et de leurs descendants qu'ils auront tôt fait de se dessaisir d'usages devenus obsolètes et contreproductifs en immigration. Observance qu'ils éprouvent comme une dette, un tribut qu'il convient de payer sous forme pratique et symbolique. Plus la détraditionnalisation et le démantèlement de l'ordre patriarcal donnent à voir leurs premiers effets en exil et plus est puissant l'appel à une tradition présentée comme intemporelle, signifiant l'ultime résistance à un changement devenu inéluctable où se décèlent les traces d'un passé révolu (Bourgois, 2001: 251-280; Guénif-Souilamas, 2000: 44-51 et 107-116). Quelques traits caractérisent ces observances apparentes et leur transgression effective. Le renversement de l'ordre des générations résulte de la plus grande maitrise des codes et rites sociaux en France par les plus jeunes et doit être contrebalancé par l'expression ostensible d'un respect subsumant ce que les parents ont perdu et ce qu'ils doivent se voir rendu par les enfants (Sayad, 1994). Renforçant ce bouleversement et procédant des mêmes effets de socialisation précoce et multiple, la « confusion des genres » au sein de la fratrie et du couple parental brouille les frontières sexuées et offre la possibilité d'investir des rôles usuellement dévolus à l'autre sexe. Cette pratique à haut risque conduit à ce qu'en contrepartie les attributs performatifs de l'hétérosexualité soient surinvestis et sursignifiés : accoutrement et hexis corporelle, pudeur et retenue selon une partition sexuée mais aussi située (à la maison ou dans le quartier, intra ou intergénérationnelle, pilosité et chevelure inversement exhibées ou dissimulées), lexiques et intonations du langage différenciés non pas tant selon une ligne de démarcation sexuée qu'en fonction de situations plus ou moins normées (dans et hors de l'institution, dans et hors de la maison, dans et hors du quartier, sous ou loin du regard des entrepreneurs 
de morale), séparation des garde-robes autant que recours à des styles vestimentaires asexués (substitution du jogging à la jupe et vice-versa), contrôle différencié et inégalitaire de la sexualité (avec les deux balises de la virginité et du viol comme nous le verrons plus loin). La contamination, l'impureté et la perte, ou du moins la crainte de celles-ci, sont donc à la fois le moteur de l'invocation de la tradition et le corollaire du processus par lequel la fiction d'une tradition continue de se dire et de tenir ses positions. Ayant depuis longtemps cessé d'être chimiquement pure, la tradition n'existe plus que par bribes, empruntant, pour un temps compté les moyens de se maintenir à tout un ensemble de ressources discursives et pratiques, de représentations plus ou moins stables. Il est dès lors possible qu'en raison même du rétrécissement du cadre normatif de la tradition, la mobilisation de ces moyens soit disproportionnée et qu'elle fasse violence aux individus auxquels elle s'impose comme à ceux qui les manipulent à des fins qu'ils ne contrôlent jamais totalement. Pris dans les contradictions d'un ordre défait par des dynamiques plus puissantes que lui, les « conservateurs » ne sont jamais complètement convaincus du bien fondé de la contrainte qu'ils exercent tout comme ceux qui en sont destinataires sont mis en position d'en douter et de contester ce qui leur est imposé. La marge, le flottement dans l'actualisation de la norme comme dans son rejet résulte du fait que ceux qui devraient l'incarner sont partie prenante d'un processus civilisateur fondé sur le doute et le libre-arbitre. Ces deux traits sont censés réaliser les conditions de la liberté, l'égalité et la laïcité, érigées en valeurs superlatives fondatrices. Pourtant, loin d'être accessibles et inscrits dans un allant de soi, ils sont l'objet d'une recherche, d'une réinscription, d'une invention perpétuelle qui contribue paradoxalement à en contredire l'intangibilité (Martuccelli, 2005 ; Roy, 2005).

Dès lors, manifester sa fidélité revêt une importance plus grande que la coutume en soi. La première est mobilisée pour que cette dernière subsiste à contre-courant. Dans l'appel exprimé de multiples manières par les aînés et souvent relayé avec des distorsions variables par les pairs, le «faire semblant» et le «faire comme si » traduisent précisément la fidélité, une façon d'être loyal sans être absolument conforme, sans attester par soi et en soi la survivance de la coutume par sa pleine intériorisation. Ainsi, toute une série de comportements et de discours traduisent le simulacre, le jeu autour et avec les règles et les signes de la coutume plus qu'une adhésion profonde à l'injonction et à sa raison. Par suite, il est possible de relier les conditions pratiques d'imposition et d'application de la norme, qu'elle soit dominante ou dominée, légitime ou illégitime au repérage des jeux goffmaniens (1973) auxquels se livrent les protagonistes pour accréditer ou discréditer les uns et les autres. Le pouvoir concédé aux entrepreneurs de morale en situation dominée n'est jamais si stable et si définitif qu'il y paraît. Cela n'atténue cependant pas la force, l'excès avec lesquels ces derniers entendent en faire la preuve, aussi dégradé et menacé soit-il. Il est même probable que l'excès dans l'expression de ce pouvoir soit le signe concomitant de son déclin face à un pouvoir normatif beaucoup plus concurrentiel, parce que mieux équipé et plus central, sur le marché de la conformation : celui de l'Etat-Nation républicain.

9 Symétriquement et contradictoirement, l'émancipation est une fin désirable pour chaque individu, mais pour les descendants de migrants, elle est toujours soumise et conditionnée au « désir » d'intégration; elle ne résulte pas des dispositions propres à chaque humain, comme à l'humanité, capable de s'adapter au milieu dans lequel il se trouve pour s'émanciper des contraintes qu'il exerce sur lui. Elle doit advenir suite à un processus de dressage et de formatage volontaire et continu. Les ultimes expressions de l'intégration normative n'ont ainsi rien perdu de leur charge institutionnelle totale 
(Goffman, 1980). Mais tout en requérant une capacité d'adaptation accrue en raison d'un contact entre sociétés et cultures encore perçu comme dangereux, l'injonction d'intégration dernier modèle repose aussi sur le désir comme pulsion de conformation. Il n'est donc pas question de faire semblant d'être intégré, en recherchant une impossible invisibilité. Il incombe aux nouveaux immigrants comme à tous ceux qui sont encore définis par leur origine de faire la preuve que les usages et les valeurs de la société « accueillante » sont connus, compris, acceptés et actualisés dans les corps et les pensées, qu'ils sont objets de désir et projetés en soi, décelables sur le corps et dans les mots. C'est sans doute pour masquer cette pulsion inavouable pour une intégration transparente derrière le désir avoué des « immigrés » de s'intégrer que le Haut Conseil à l'Intégration (HCI) s'est avisé d'élaborer une doctrine en matière d'immigration et de gestion des minorités. Dans ses avis successifs tout comme dans les questions dont il s'est saisi persiste un fil rouge, celui de l'asymétrie entre l'Etat accueillant et l'être accueilli, entre la puissance publique et la malléabilité attendue des immigrants, futurs citoyens pour certains d'entre eux. Vus comme n'ayant aucune expertise sur le pays dans lequel ils arrivent, les immigrants, qui, même devenus Français, le restent souvent toute leur vie, sont sommés de faire la preuve qu'ils ont bien compris ce qui est attendu d'eux et éventuellement toléré pour que, cette condition pré-requise étant remplie, ils puissent éventuellement voir légitimer la réalisation et l'actualisation de certaines attentes et aspirations. Si l'horizon d'attente de tous les individus légitimes dans les sociétés occidentales démocratiques et égalitaires tend à rejoindre celui de l'Etat censé leur permettre de s'en rapprocher, tel n'est pas le cas pour les immigrants et leurs descendants. Définis par un statut illégitime, même s'ils contribuent pourtant à alimenter des aspirations plus souvent convergentes que divergentes avec les membres de la société où ils vivent et à finir par leur ressembler par bien des aspects, ils ne sont pas crédités d'une même aspiration à la liberté et à l'émancipation. Il convient donc de la faire advenir en eux, en lieu et place d'un attachement à « la » tradition considérée comme persistante et nuisible. Et cela reposerait sur le « désir » d'intégration alors que le respect de la tradition ne peut être mobilisé qu'en tuant tout désir. Il y aurait comme de la libido dans l'intégration, pulsion supposément dissoute par la corrosion de la tradition. La valorisation de ce qui est désirable plus encore que de ce qui est raisonnable se comprend comme l'emprunt, aux normes d'individuation devenues dominantes, d'un ressort qui ne conduit cependant pas toujours à la réalisation de soi mais plus sûrement à la conformation sous contrainte. Ainsi, le désir d'intégration ne mène-t-il lui aussi qu'à un simulacre, un faux-semblant qui commande les mises en scènes auxquels se livrent les impétrants sous le regard de leurs juges comme les vérités statistiques -fondées, faut-il le rappeler sur les déclarations des personnes enquêtées et aucunement sur l'observation de leurs pratiques ou sur l'exploration raisonnée de leurs motivations- veulent le montrer. Cependant, cette embardée sur le terrain accidenté du discours psychanalytique en dit plus sur ceux qui le mobilisent en faveur de la norme intégrationniste que sur ceux qui doivent en manifester la preuve sous peine d'être considérés comme des sujets aliénés et souffrants.

L'objectif de conformation des deux assignations dénote un parallélisme qui se prolonge ici dans la mobilisation en vue d'y parvenir, du désir, sentiment et émotion éminemment contemporains, pris entre subjectivité et normativité. Le conservatoire de la coutume et l'impératif catégorique d'intégration sont à la fois antinomiques et présentés selon les mêmes registres discursifs : une aspiration légitime et indiscutable, offrant plénitude et épanouissement individuel. Les deux en appellent à l'authenticité, 
à la réalisation de l'individu tout en opérant selon des principes normatifs qui aiguisent leur concurrence et réduisent la marge de manœuvre individuelle voire la prennent en étau. Leur normativité contredit la tendance à l'adhésion fondée sur la reconnaissance libre des désirs et sur la construction des attachements et des appartenances. L'imposition paradoxale de l'une et l'autre assignation ne peut se faire qu'au prix du déni d'attentes qui ne deviennent viables et acceptables qu'en revêtant la forme et la rhétorique prescrites par les entrepreneurs de morale. Se déguiser, devenir à tout prix invisible pour passer inaperçu et aller de l'avant. Seul le dicible doit se dire et les descendants de migrants mesurent les limites discursives et existentielles de leur affirmation dès lors qu'ils sont sommés de s'expliquer sur les motifs de leurs choix et de leurs actes. L'attente tenue pour légitime par les entrepreneurs de morale de choix loyaux porte dès lors sur les aspects les plus intimes de l'existence. Or, tout en appartenant à l'espace d'édification de normes morales et juridiques, ces expressions de l'intime s'en sont depuis un demi-siècle autonomisées et graduellement émancipées en se revendiquant comme sécularisées et privées: présentation de soi et accoutrement, sexualité, orientation sexuelle, sentiment amoureux et désir, mariage ou union libre, fécondité et contraception. Ce sont ces registres d'affirmation et de conformation, situés à la jonction entre collectif et particulier qui sont tout particulièrement investis, ainsi que les corps qui les incarnent, par la volonté de puissance d'instances hiérarchisées dans le champ symbolique: d'un côté, la Nation républicaine et les défenseurs de sa pleine légitimité, de l'autre la famille illégitime, dite à problèmes, sous ses nouveaux dehors : musulman et immigré.

11 L'invisibilité est d'autant plus de mise pour les individus soumis à ces injonctions qu'elles se présentent comme antinomiques et proposeraient des modèles diamétralement opposés et irréconciliables: ces Français d'ascendance migrante doivent laisser croire qu'ils sont convaincus de l'irréductibilité de l'une à l'autre et de l'inéluctabilité du choix. Une telle alternative serait recevable si l'une comme l'autre n'opéraient pas pratiquement en rappelant ceux auxquels elles s'adressent à une même différence, entendue comme un trait saillant indélébile et irréductible qu'il convient pour l'une d'entretenir et pour l'autre d'éradiquer.

12 Si pour l'une il s'agit de constituer les descendants comme le conservatoire d'une différence qui pour être essentialisée n'en est pas moins réinventée et réinterprétée en immigration, il s'agit pour l'autre d'extirper ces mêmes descendants d'une différence à laquelle ils sont, ipso facto, rappelés, et contre laquelle ils sont mis en garde, parce que son assise traditionnelle les empêcherait de devenir des individus libres. De ces ressemblances surgit un autre paradoxe : c'est en raison même des ressorts communs de leur logique d'action, la conformation, que ces injonctions se disqualifient mutuellement et sont aveugles à leurs similitudes et leur convergence. Considérant ce paradoxe, il est possible de conclure que les raisons de leur aveuglement partagé procèdent de leur logique concurrentielle. Elles visent à rallier les mêmes troupes à un dessein apparemment opposé : la validation et la viabilité d'un modèle. Dans un cas, survivance d'une tradition réinventée après avoir été défaite et rompue par la première modernité, celle de l'occidentalisation et/ou du colonialisme. Dans l'autre, la pérennité d'un modèle intégrateur dont l'élan assimilationniste s'est heurté aux "petites patries » puis au démenti de la décolonisation et de la désintégration postindustrielle. De ce parallélisme se dégage un dernier argument: il n'y a aucun hasard dans le fait que les ultimes comptables de ce modèle, ceux qui doivent journellement faire la démonstration de sa postérité et sa vertu soient les descendants de ceux qui en 
connurent les vertus modernisatrices jusqu'à la déstructuration (Sayad, 1992) : les paysans colonisés devenus des ouvriers immigrés dans leur ancienne métropole et aujourd'hui leurs descendants, les Français d'ascendance migrante et coloniale. Enfin, derrière ce parallélisme se profile un décalage: la socialisation de ces nouveaux Français est exercée sous contrainte normative, discernant dans leur comportement et leurs propos les signes d'adéquation et les marques de loyauté, alors que les socialisations de leurs contemporains prônent l'égalitarisme, par la négociation et le contrat et réservent le régime de contrainte littéralement à la marge, aux déviants. De sorte que tout descendant de migrant serait potentiellement un déviant, se tiendrait à la marge, tant qu'il n'a pas fait la démonstration de son adaptation, de sa conformation au système normatif dont les critères prennent à son attention un tour univoque et exclusif. Toutes preuves qu'il ne pourra pas fournir. Pris en défaut, il autorise par là l'expression raciste, l'acte discriminatoire, en fournit la justification et en procure l'absolution. Il est seul responsable du rejet subi, aussi bien dans le registre de la trahison à l'égard des ascendants et des pairs que dans celui de la loyauté bafouée à l'égard de la République. Sans doute faut-il voir là pourquoi leurs entrepreneurs de morale respectifs sont ce que Becker appelle des "croisés ». En renvoyant à un régime révolu, elles les assignent doublement: à une différence censée manifester les résurgences d'un ordre archaïque dans une modernité occidentale encore englobante et à une structuration identitaire unifiée et univoque qui n'existe plus guère que parmi les générations de la société industrielle disparue. Précisément celle des immigrés et des ouvriers aux destinées communes dans un monde tendu vers le même horizon. Mais certes pas celle de leurs enfants qui eux sont contemporains des incertitudes identitaires, des destins mis en concurrence et des horizons démultipliés.

\section{Sexuation partagée et départagée}

13 Si ces assignations censées s'opposer partagent le même sens normatif, attestant le même mode d'action et d'imposition, leur procédure de sexuation et l'usage qu'elles font des identités ainsi sexuées revêtent des contours différents tout en s'appuyant sur les mêmes figures naturalisées du féminin et du masculin. Apparemment, l'intégration par émancipation et l'appel à la fidélité coutumière ne répondent pas à une même conception de la partition sexuée tout en continuant à invoquer l'une comme l'autre l'existence d'identités sexuées socialement et culturellement différenciées. À en croire certains observateurs, elles marquent la ligne de partage entre une tradition entretenant un régime patriarcal et sa justification de l'asymétrie entre les sexes et une modernité actualisée par un régime démocratique fondé sur l'égalitarisme abstrait, aveugle aux différences sexuées. Elles ne reposent pas, du moins en première analyse, sur les mêmes définitions de la sexuation. L'une appelle à la défaite du genre, l'autre entend le pérenniser; l'une prétend «dissoudre la hiérarchie » en se fondant sur la révolution anthropologique de la dénaturalisation de la procréation et de la privatisation du désir et du plaisir, l'autre plaide pour la complémentarité fondée sur la nature biologique des deux sexes et sur l'ordre immuable du pouvoir des hommes sur le ventre et la sexualité des femmes. Ainsi cette dernière en appelle à une nature féminine inaltérable fondée sur la maternité, là où l'autre s'inscrit dans la lutte pour l'émancipation des femmes de leur nature de génitrice. Si l'une naturalise, l'autre dénaturalise; si l'une magnifie une essence, l'autre en dénonce les effets aliénants. Et ceci vaudrait autant pour les femmes que pour les hommes. Ce serait donc bien en 
raison de cette divergence d'interprétation et de définition des statuts du féminin et du masculin que les assignations constituant l'injonction paradoxale, fidélité coutumière et intégration par émancipation, ne servent pas les mêmes objectifs normatifs et sont en conflit quant au sort fait aux femmes.

Pourtant, dans ce moment particulier du passage d'une modernité à l'autre, les deux injonctions revêtent des contours similaires. Ainsi, en convoquant la nature, la différenciation sexuée revêt la même forme dans les deux entreprises de conformation. En effet, l'une comme l'autre réassignent à une nature sexuée les individus qu'elles prétendent conformer, en s'appuyant sur les mêmes ressorts biologiques différenciés et essentialisés. Loin de la défaire au nom d'un égalitarisme abstrait, l'injonction à l'émancipation prend appui sur la figure de la femme génitrice et éducatrice pour instaurer une rupture intergénérationnelle et ainsi en investir les mères tout en prétendant en libérer les filles. Le dispositif réussit le tour de force de simultanément renvoyer toutes les femmes prises dans une définition migratoire de leur destin, à une origine essentialisée et induire une concurrence entre mères, forcément prisonnières de la tradition, et filles qui ne peuvent que vouloir s'en libérer. Elle fait exister, à la marge et en rupture avec les normes dominantes, une certaine nature féminine qui ne saurait être extirpée que par la civilisation des filles contre leurs mères. Cependant, cette entreprise de conformation emprunte des voies paradoxales lorsqu'elle prétend libérer les Françaises d'ascendance migrante et coloniale en réinvestissant leur corps d'un éternel féminin, une féminité qu'il leur faut magnifier pour prouver leur intégration et arborer contre des hommes soupçonnés de vouloir les en déposséder pour les asservir. Leur outil de libération ne consiste pas à se dés-assigner d'une «nature " féminine intrinsèquement définie par des rôles sexués inscrits dans une matrice patriarcale, fut-elle dé-traditionnalisée, mais à en signifier les traits les plus pérennes en incarnant l'amante, objet de désir, la femme, disponible sur le marché matrimonial, la mère, éducatrice des hommes.

Ces mêmes hommes, pères, frères, parents, qui investissent la féminité de

leurs femmes selon un registre lui aussi naturalisateur, invoquant la complémentarité fondée sur une différence naturelle des sexes pour les ré-assigner à une place coutumière dont la défaite est pourtant consommée par la migration : réceptacles d'une sacralité centrée sur la virginité, gardienne d'une fécondité endogame et d'une éducation conservatoire du patriarcat. Cette égale mobilisation de la féminité indique qu'il n'y a pas rupture avec la définition patriarcale de la femme, ni dans le type d'émancipation cadré et contrôlé par une idéologie normative proposée aux descendantes d'immigrants nord-africains, ni dans le refus de toute dialectique entre émancipation subjective et fidélité coutumière qu'affirment des entrepreneurs de morale, prisonniers de leur nostalgie et jaloux de leur pouvoir. L'éternel féminin continue d'être le lot des femmes et tout particulièrement de celles qui sont visiblement différentes. Tout en devant se défendre d'être prises au piège de différences ethniques, religieuses, culturelles qui viennent opportunément oblitérer les inégalités sociales ou les ségrégations spatiales réelles qu'elles subissent, les Françaises d'ascendance immigrée sont condamnées à en actualiser les traits dans leur corps et dans leur manière d'être et d'agir. L'intégration par émancipation repose presque exclusivement sur les femmes. Elles ne peuvent occuper la place qui leur est réservée qu'en se conformant au rôle qui leur est dévolu : incarner la variante post-migratoire de l'éternel féminin. Ainsi, hommes et femmes existent bien en immigration, et ils 
continuent d'exister après, lorsqu'il s'agit pour eux d'accéder à une place viable dans la cité politique. Les fonctions différenciées en régime migratoire se déplacent sans disparaître en régime intégrationniste. Pas plus que la fidélité coutumière ne s'impose et ne s'exerce hors de toute définition sexuée, la preuve de l'intégration par émancipation ne s'administre en étant aveugle aux natures sexuées des impétrants. À l'inverse, les deux conformations concurrentielles composent une alchimie complexe qui se sédimente selon des procédures parallèles. Au terme de longues manipulations, indépendamment de ceux qui les opèrent, se dépose une nature binaire, sexuée, masculine et féminine, aux ressemblances troublantes, aux vices et aux vertus symétriques. En effet, l'appel à la norme des uns repose sur la disqualification de ce qui pour les autres apparaît comme hautement valorisé. Le respect dû aux aînés, la solidarité intergénérationnelle, la pudeur vestimentaire, la retenue verbale, l'abstinence sexuelle, la place faite aux croyances, sont autant de règles de conduites tenues pour éminemment contraires à la promotion de l'individu libre et affranchi de règles pouvant le contraindre. Valorisées par les tenants de l'émancipation, celles-ci se nomment: le respect sous condition de réciprocité, l'autonomie, le libre-arbitre, l'autorité, la liberté de disposer de son corps et de sa sexualité, la civilité. Sans se correspondre terme à terme, ces différents registres indiquent la superposition des systèmes normatifs que des conflits exacerbés opposent en désajustant les termes qui les composent. Ainsi la liberté sexuelle n'est pas contredite par la pudeur vestimentaire, le respect sous condition de réciprocité ne contredit pas la solidarité intergénérationnelle. L'autonomie n'abolit pas les liens et attachements de tous ordres que revendiquent ou dont s'accommodent les individus.

Dès lors, un des effets les plus flagrants de l'opposition concurrentielle entre les deux injonctions normatives aura été, contre toute vraisemblance, la promotion d'une fiction selon laquelle la partition sexuée se jouerait non plus universellement, entre hommes et femmes, mais sectoriellement, entre deux civilisations ou selon une rhétorique encore plus restrictive entre deux cultures. Les individus affranchis de leur origine sociale, ethnique et de leur statut économique ne subiraient plus les effets pérennes d'une assignation à leur nature sexuée. En revanche, la vraie partition sexuée séparerait deux cultures, deux civilisations, l'une étant presque parvenue à s'affranchir du patriarcat et de ses survivances inégalitaires et sexistes et l'autre dépositaire constant de l'ultime forme archétypale du genre, celle qui asservit les femmes aux hommes, "sans distinction de classe, de race ou de condition sociale", mais en soulignant la ligne de démarcation entre « eux » et « nous ». La preuve que la tradition est bien là serait administrée par les femmes et l'oppression qu'elles subissent. La forme revêtue par les débats relatifs aux migrants, à leurs descendants et à leur visibilité est inédite sinon inouïe. Elle consiste à appliquer la catégorie du genre (Delphy, 2001) à l'analyse des rapports sociaux entre genres et générations postmigratoires et postcoloniaux pour en déduire que le genre aurait plus de capacité d'élucidation dans l'enclave où sont artificiellement regroupés ces nouveaux protagonistes d'une "francité » politiquement dérangeante que dans le reste de la société d'où seraient éradiqués les signes d'inégalité et d'oppression. La tentation d'ethniciser le genre ou de "genrer " certaines minorités ethniques point sous plus d'un énoncé ou d'un mot d'ordre sans que soient rappelées les précautions qu'impose l'usage de ce type de catégorie. 


\section{Variations et atténuations individuelles des pressions normatives}

17 Le propre de l'individualisme contemporain réside dans le fait inédit et central qu'à toute volonté de soumettre à une norme, même dominante, est opposable l'invocation de la légitimité de l'individuation, du respect de l'intégrité et de la liberté de chacun, que celle-ci soit négative ou positive. Ce processus atteste d'une croissante volonté de disposer de soi que résument deux problématiques propres à la modernité tardive, celle de «la propriété de soi » (Castel, Haroche, 2001) et celle de "l'invention de soi» (Kaufman, 2004), témoignant toutes deux d'une «consistance du social » renouvelée (Martuccelli, 2005). Si l'on accepte l'analyse de ce dernier selon laquelle le social n'a pas perdu sa consistance mais a vu celle-ci changer, devenant « élastique » et « décalée », il semble que s'inventer en tant qu'individu tout en s'efforçant de préserver une propriété de soi durement concurrencée soit une entreprise plus insurmontable pour les Français d'ascendance immigrée. En effet, leur qualité d'individu est soumise à condition en raison de la double injonction à la conformation à laquelle ils doivent se plier, il leur est plus difficile qu'à d'autres de revendiquer des registres d'invention de soi n'obéissant pas aux normes implicites qu'ils doivent actualiser. Ainsi, pour ces derniers, il s'agirait de commencer paradoxalement là où s'arrêtent les individus "sans origine »: là où ceux-ci sont exemptés d'une parfaite adéquation à la norme, ceux-là doivent incarner sa toute puissance.

En dépit de cette pression normative, "ces individus sous condition " esquissent un espace de réflexivité (Beck, 1986, Giddens, 1989) où se jouent accommodements, négociations, détournements, abandons et confrontations aux normes. Ce jeu investit tous les interstices entre les individus et les institutions aux contours et aux assises de moins en moins stables et pérennes. La famille en France qu'elle soit immigrée ou pas ne repose plus aujourd'hui sur des fondements institués, unifiés, immuables et capables de résister aux transformations introduisant de la plasticité et de l'incertitude dans un monde social jusqu'alors défini par des règles massives et durables. À l'instar de celles des Français "d'origine », les attitudes des Français d'ascendance migrante et coloniale ne peuvent se lire qu'à la lumière de la recomposition réflexive des relations entre individus. De même, leur capacité à atténuer les pressions normatives dépend largement des ressources plus ou moins disponibles qu'ils peuvent mobiliser au regard de configurations familiales, scolaires, sociales et politiques plus ou moins stables mais aussi du jeu introduit dans la dialectique des rapports sociaux qu'ils expérimentent. S'ils ne peuvent pas amortir seuls les tensions relationnelles qu'ils subissent, ils ne peuvent pas moins tenir à distance les messages normatifs qui s'imposent à eux en ordre dispersé. Cette superposition décalée entre niveau interpersonnel et niveau institué explique la pesanteur éprouvée par les individus assignés mais aussi les marges de manœuvre que ces mêmes individus se ménagent et consolident ici et là, au détour d'un rappel à l'ordre trop direct ou d'un principe de loyauté trop massivement assené.

Or, le statut d'illégitimité (Sayad, 1999) ou de subalterne (Spivak, 2004) est l'élément nécessaire à une lecture précise des rapports entre ces Français d'un nouveau type et leurs entrepreneurs de morale ou d'identité. Il est l'étalon qui permet de mesurer, selon les personnes mais aussi pour une même personne, selon les circonstances, la plus ou moins grande capacité à se distancer voire à invalider la norme énoncée. En effet, ils sont tenus pour des individus illégitimes tant qu'ils n'ont pas fait la preuve de leur 
loyauté, et ce quels que soient leur sexe et leur position au sein de la fratrie. Ils sont donc d'emblée assignés à une position dominée à partir de laquelle ils font montre de capacité à se plier ou à résister aux normes qui s'imposent à eux. Pour autant, cette résistance à des injonctions contradictoires et anachroniques les confronte au risque cumulé d'une autonomisation à l'égard de normes obsolètes ou exorbitantes sans régime adéquat de protection de soi et d'autre part une dépendance subie, et non choisie, qui ne leur offre que peu d'accommodement entre leurs aspirations et celles qu'expriment les leurs. Vraisemblablement, filles et garçons ajustent leurs attitudes et leurs choix aux situations plus qu'ils ne les mettent en conformité avec un dessein précis et conscient. Dès lors, l'impression d'observer des comportements incohérents ou ambivalents, ne traduisant aucune logique interne, conduit trop hâtivement à les rabattre sur leur dimension spectaculaire et outrancière pour n'en dégager qu'un sens propre et unifié, délaissant le sens figuré, re-signifié et souvent pluriel, que chaque individu attribue aux expériences qui le façonnent et aux traits qui le rendent visible.

\section{Le mariage et ses biens : un marché concurrentiel}

Un seul exemple suffirait à illustrer cette oscillation entre obéissance et transgression : les multiples manières de s'insérer sur des marchés amoureux et matrimoniaux aux frontières étanches, le choix du conjoint à la française ou le mariage forcé selon une appellation devenue contrôlée. Le mariage est devenu le signe ultime et suprême de conformation qui conduit filles et garçons à développer des stratégies souvent parasitées par les effets incontrôlables de l'adhésion aux codes et rites amoureux adolescents. Se présenter dans le rôle du rejeton soucieux de fidélité coutumière puis dans celui du candidat à une intégration émancipatrice n'épuise pas les possibles et livre des parcours oscillants. Les voici donc passant d'un registre à l'autre selon les circonstances et les interlocuteurs et offrant des mises en scène paradoxales et contradictoires dont on ne saurait conclure si elles visent à rassurer les entrepreneurs de morale en gagnant du temps ou si elles traduisent une véritable adhésion à l'une ou l'autre des options imposées. Les récits traduisent une discontinuité délibérée où se succèdent, se superposent et se contredisent docilité filiale, révolte sourde contre le destin imposé, déni de ses propres aspirations et désirs, adhésion éperdue aux standards de l'électivité amoureuse, incrédulité devant ces mêmes standards et revendication d'usages censément obsolètes et oppressifs re-signifiés pour un usage unique.

21 Par exemple, la revendication par les filles de la virginité ne signifie pas qu'elles se résolvent à se réserver pour un époux choisi par d'autres, ce qui accréditerait l'hypothèse de l'identité entre virginité et mariage forcé. Elle signifie tout autant que, sur un marché amoureux encombré, elles y voient un signe de distinction et un signal de sélection d'un partenaire attentif et compréhensif, ce qui consolide l'hypothèse de la multiplication de règles subjectives régissant les transactions amoureuses et sexuelles. L'une et l'autre de ces interprétations ne signifient pas en soi l'écart à la tradition ou à la modernité mais elles illustrent, ensemble, les formes d'articulation inédites qu'opèrent les jeunes filles entre volonté de contrôle de leur sexualité et liberté sexuelle personnelle, entre conduites hétéronomes et décisions individuelles. De même, les garçons qui se soumettent à la décision de mariage des parents ne sont guère plus consentants que les filles, mais ils opèrent des arbitrages entre possible et impossible. 
Prenant acte du fait que leur est fermé un marché matrimonial local où ils ne sont guère prisés, ils s'ouvrent à un marché " délocalisé » où ils joueront d'atouts jusqu'alors méprisés : docilité normative, fidélité coutumière, faible mobilisation de stratégies de séduction au rendement aléatoire, respect des aînés, attitude dominatrice et protectrice, identité hétérosexuelle affirmée. La partition sexuée et sexuelle structure fortement le champ matrimonial pour deux raisons apparemment antagoniques mais qui en réalité interagissent profondément. D'une part les parents, vus comme dépositaires d'une tradition figée, subissent les effets d'optique imposés par un monde en constante transformation et accélération et une expérience individuelle saisie par ce rythme. Le désajustement des temporalités accrédite l'idée qu'ils imposent leur raison patriarcale intacte alors qu'elle est suspendue à des incertitudes durables dont elle subit les inflexions et les turbulences. D'autre part, la culture dominante édicte des règles de partition entre les descendants de migrants pour les faire entrer sur des marchés séparés où ils doivent simultanément exprimer leur désir du contact entre «races » et le réaliser sous sa forme la plus euphémisée et policée, donc la moins visible. Cette règle implicite mais puissante conduit à une hiérarchisation de ces prétendants à "la naturalité française» selon leur sexe: les filles sont fortement valorisées en dépit ou en raison du fait qu'elles ont un accès contrôlé à une union exogame, dans le même temps, les garçons sont d'autant moins valorisés qu'ils ont un plus grand accès au marché de l'exogamie tout en n'y étant pas les bienvenus. Dans les deux cas, la hiérarchie qui prévaut ici repose sur des stéréotypes racistes et sexistes hérités de l'époque coloniale et largement métabolisés dans les représentations et usages des immigrations postcoloniales. Si alors, la figure du colonisé représentait la quintessence d'un exotisme subalterne pouvant être soumis sans scrupule, son ombre portée entrave aujourd'hui l'exercice d'une liberté pourtant associée à la société élective logiquement interdit aux figures les plus aisément associées au monde colonial et à son ordre inégalitaire. La conception d'une ségrégation appliquant des règles de contact sous contrôle n'a pas seulement conduit à sa mise en œuvre spatiale, aux quartiers ségrégés où vivent des communautés supposées repliées sur elles-mêmes mais aussi à son impression dans les corps hautement sexualisés des Français d'ascendance migrante et coloniale par l'exercice d'un contrôle de leur sexualité. Ainsi, si des parents et leurs alliés objectifs, entrepreneurs d'identité, s'acquittent trop bien du contrôle de la sexualité de leurs filles sans toujours pouvoir lui trouver les justifications qu'impose le régime individualiste contemporain, faisant ainsi d'eux des suspects en puissance, la sexualité des garçons n'est pas pour autant laissée libre de s'exprimer. Elle doit présenter simultanément tous les signes d'une hétérosexualité explicite et toutes les garanties d'un comportement policé. Son contrôle procède d'un cercle plus large et plus étanche où les parents destitués peuvent être efficacement secondés ou remplacés par des opérateurs multiples formant des alliances parfois paradoxales : relais d'opinion, personnels de l'éducation nationale, agents de la police, prédicateurs, personnels de sécurité. Il est frappant d'observer que ce cercle se compose variablement mais de façon croissante de personnes elles-mêmes définies par les origines tenues en suspicion, où les videurs de boîte de nuit prompts à appliquer les règles sélectives d'une ségrégation festive s'avèrent les parfaits supplétifs de prédicateurs sourcilleux sur la moralité de leurs fidèles et vice versa. Dans les deux cas, la frontière ethnique entre "nous » et " eux » est scrupuleusement gardée. L'illusion d'une morale sexuelle fondée sur la liberté et le consentement fait ainsi long feu dès lors que l'on y discerne une norme sexuelle qui prétend accréditer l'idée que les filles 
seraient naturellement portées vers une sexualité revêtant tous les traits du commerce amoureux occidental alors que les garçons resteraient prisonniers de leur penchant naturel pour la volonté de puissance et la sexualité brutale et insensible qui s'exécute dans et hors du mariage. Dès lors, leur présence sur des marchés matrimoniaux séparés se justifie pleinement puisqu'il s'agit en quelque sorte de protéger les filles de leurs trop proches prédateurs en leur offrant l'accès à un marché de transactions présentées comme plus policé. Mais en croyant les soustraire à l'étouffoir d'un monde censé être sans amour, le discours normatif sur les affinités électives contribue à leur renvoi vers des contraintes par corps que traduisent deux options : le mariage imposé dans le pays d'origine des parents, l'union avec un conjoint choisi dans le pays des parents et «importé » en France dans le cadre d'un regroupement familial. Il est d'ailleurs probable que les chiffres indiquant annuellement le nombre de rejoignants concernent essentiellement des Français d'ascendance immigrée qui ont épousé des ressortissants du pays des parents. Cette pratique constitue alors vraiment un « mariage mixte » dans la mesure où les conjoints n'ont pas vécu dans le même pays, n'ont pas partagé les mêmes socialisations et ne partagent peut-être même pas une commune conception de la vie amoureuse, du mariage et de la sexualité. Le fait qu'ils n'aient pas la même nationalité traduit une distance existentielle d'autant plus grande qu'elle résulte de la segmentation du marché français. En se voyant imposer une séparation par corps, certains Français d'ascendance immigrée trouvent une alternative dans l'endogamie en France, qui n'est rien d'autre que l'homogamie sociale analysée par Bozon et qui serait en l'occurrence appliquée aux quartiers ségrégés, ceci permettant d'éviter d'autres mariages qui n'ont plus rien d'homogame et n'ont que l'apparence de l'endogamie, ceux avec un conjoint ou une conjointe choisi au pays des parents. Ainsi, même avec son cortège de renoncements, de désillusions, de compromis, l'observation du champ amoureux et matrimonial témoigne des déplacements et des tensions qui le traversent et de l'ingéniosité dont peuvent faire preuve les jeunes qui doivent inéluctablement si investir. La complexité du paysage des sentiments offre une illustration de la difficulté à se situer entre deux appels normatifs qui n'entendent pas se fondre et moins encore se confondre. Dès lors, les jeunes en question opèrent des hybridations, des combinaisons qui ne peuvent être lues que comme autant de trahisons. Tout se passe comme si ce monde croissant de l'à-côté de la norme ne pouvait qu'être récusé et invalidé par des entrepreneurs de morale qui ne savent comment s'accommoder de ces pratiques incertaines et difficilement étiquetables sinon en les repoussant hors de leur horizon des possibles. Il n'est guère surprenant que ces horizons soient désajustés et n'aient que peu de point d'intersection, sinon au prix d'une ingéniosité performative (Butler, 2005) et d'une capacité à donner le change, aptitude inégalement distribuée entre tous les protagonistes.

\section{Disposer de soi, une entreprise incertaine}

Si l'on accepte l'idée que les individus contemporains sont pris dans une injonction entre l'invention de soi en régime concurrentiel et la fatigue d'être soi qui en est le corollaire (Kaufman, 2004 ; Ehrenberg, 1998), la volonté de disposer de soi est d'autant plus contrariée que s'y opposent des vicissitudes et des aléas propres à l'individualisation. Que ceux-ci soient qualifiés dans des registres sexuels, matrimoniaux ou religieux n'est somme toute qu'une figure de style qu'il convient de déjouer pour montrer qu'en définitive, ce qui se joue là est la capacité à disposer de soi, 
non seulement en tant que personne mais aussi en tant qu'acteur intime, économique, social, politique et ultimement symbolique. Dès lors, les Français d'ascendance migrante et coloniale seraient indisponibles à eux-mêmes non pas en raison de la pesanteur de traditions dont on a montré qu'en régime d'illégitimité, elles sont profondément affectées et reformulées par l'immigration mais du fait de l'hypothèque identitaire et symbolique qui fait d'eux des citoyens sous condition. Contrairement aux apparences, ce statut hypothéqué ne l'est pas moins pour les filles que pour les fils de ces immigrants qui partagent le stigmate cumulé d'être arabe et musulman, deux dimensions constitutives du rapport de familiarité, entre attirance et de rejet, entretenu avec les descendants du prolifique indigène de l'empire colonial français venu en métropole. Or, ce même rapport de domination qui fut l'objet d'une sexuation ambiguë dans l'ordre colonial, permettant la prédation et la soumission des deux sexes, est sexuellement différencié et hiérarchisé aujourd'hui dans la France postcoloniale (Guénif-Souilamas et Macé, 2004). Le patriarcat étant voué à se dissoudre dans la vulgate intégrationniste, en trahir les dernières expressions tombe sous le coup d'une condamnation sans appel, prononcée par ceux qui prétendent protéger, y compris contre elles-mêmes, les filles d'immigrants arabes et musulmans. Ainsi, celles-ci sontelles créditées favorablement, en ce qu'elles seraient "naturellement" enclines à vouloir échapper au contrôle qu'exercent sur leur corps et leur sexualité des hommes «naturellement " portés à vouloir les dominer. Au fondement de cette disposition favorable à l'égard du sexe "faible » dès lors qu'il est d'une certaine origine, se trouve un postulat que les théories de la domination masculine et du patriarcat se sont employées à déjouer comme étant le ressort même de la domination : sa naturalisation par un ensemble de mécanismes culturels, politiques, symboliquement justifiés (Delphy, 2001). Appliqué aux descendants de migrants, ce postulat conduirait à penser que c'est en raison même de leur incapacité à se déprendre de leur nature que ces hommes dominent avec autant d'aplomb, d'aveuglement et de persévérance leurs femmes. Alors que c'est précisément en raison d'un "processus de civilisation " singulier (Elias, 1969), mixant des logiques patriarcales et tribales à des justifications religieuses, voire théologiques, toutes deux combinées avec une expérience migratoire soumise à la normativité dominante française, que se rejouent et se recomposent les rapports de genre et de génération. Dans ce processus constant de réévaluation, il n'y a pas trace de régression, par contre une logique de confrontation inégalitaire y est décelable, qui génère autant de crispations que de curiosité ambiguë envers une culture dont les critères de domination n'ont jamais été aussi brutalement et frontalement remis en question. Cette remise en question n'est pas le fait d'une seule autre culture, commodément dénommée arabo-musulmane, mais d'une pluralité de revendications et d'affirmations culturelles qui toutes affaiblissent la conception unifiée et unifiante de la norme ethno-nationale (Lorcerie, 2003; Bourcier, 2005). Celle-ci s'appliquant idéalement et prioritairement aux immigrants et à leurs enfants, autrement dit à l'Autre, c'est parmi les premiers puis les seconds que sont désignés ses ennemis "naturels ", ceux qui ne se prêtent pas volontiers au jeu de la conformité béate. Les plus visibles d'entre eux étant les Français musulmans ou leurs doubles, les jeunes Arabes des quartiers disqualifiés (Guénif-Souilamas et Macé, 2004), ils sont prioritairement destinataires de sommations à se conformer et simultanément destitués de toute légitimité à incarner cette même conformité érigée en mythe identitaire. Un mythe sexualisé, hyper-sexualisé pourrait-on dire, pour n'en être que plus inaccessible. 


\section{BIBLIOGRAPHIE}

BANCEL Nicolas, BLANCHARD Pascal et LEMAIRE, Sandrine, éd. (2005) La fracture coloniale, Paris, La Découverte.

BECK Ulrich $(1986,2001)$ La société du risque, Paris, Aubier.

BECKER Howard $(1963,1985)$ Outsiders, Paris, Métailié.

BOURCIER Marie-Hélène (2005) Sexpolitiques, queer zone 2, Paris, La Fabrique.

BUTLER Judith $(1990,2005)$ Trouble dans le genre, Paris, La Découverte.

BOURGOIS Philippe $(1995,2001)$ En quête de respect, Paris, Le Seuil.

CASTEL Robert et HAROCHE Claudine (2001) Propriété privée, propriété sociale, propriété de soi, Paris, Fayard.

DELPHY Christine (2001) Penser le genre 2, L'ennemi principal, Paris, Syllepse.

DUBAR Claude (2000) La crise des identités, Paris, PUF.

EHRENBERG Alain (1998) La fatigue d'être soi, Paris, Odile Jacob.

ELIAS Norbert (1969) La civilisation des mœurs, Paris, Calmann-Lévy.

GIDDENS Anthony $(1989,1994)$ Les conséquences de la modernité, Paris, L’Harmattan.

GOFFMAN Erving (1968) Asiles, Paris, Minuit.

GOFFMAN Erving (1973) La mise en scène de la vie quotidienne, la présentation de soi, Paris, Minuit.

GÖLE Nilüfer (2005) Interpénétrations. L'islam et l'Europe, Paris, Galaade Éditions.

GUÉNIF-SOUILAMAS Nacira (2000) Des « beurettes » aux descendantes d'immigrants nord-africains, Paris, Grasset.

GUÉNIF-SOUILAMAS Nacira (2002) Immigration-intégration, le grand découplage, Ville, école, intégration, $\mathrm{n}^{\circ} 131, \mathrm{CNDP}$.

GUÉNIF-SOUILAMAS Nacira et MACÉ Éric (2004) Les féministes et le garçon arabe, La Tour d'Aigues, L'Aube.

HÉRITIER Françoise (2002) Penser la différence, dissoudre la hiérarchie, Paris, Odile Jacob.

KAUFMAN Jean-Claude (2004) L'invention de soi, Paris, Armand Colin.

LAPEYRONNIE Didier (1993) L'individu et les minorités, Paris, PUF.

LATOUR Bruno $(1991,1993)$ Nous n'avons jamais été modernes, Paris, La Découverte.

LEPOUTRE David (2005) Mémoires de familles immigrées, Paris, Odile Jacob.

LORCERIE Françoise dir. (2003) Le défi ethnique, Paris, ESF-INRP.

MARTUCCELLI Danilo (2005) La consistance du social, Rennes, PUR.

RANCIÈRE Jacques (1981) La nuit des prolétaires, Paris, Fayard.

ROY Olivier (2005) La laïcité face à l'islam, Paris, Stock.

SAYAD Abdelmalek (1991) L'immigration, les paradoxes de l'altérité, Bruxelles, de Boeck.

SAYAD Abdelmalek (1999) La double absence, Paris, Le Seuil. 
SPIVAK Gayatri Chakravorty (1985) Can the Subaltern Speak? Speculations on Widow-Sacrifice, Wedge, 7/8, Winter/Spring.

SPIVAK Gayatri Chakravorty (1999) Acritique of postcolonial reason, Cambridge, Mass., Harvard University Press.

STORA Benjamin (1999) Le transfert d'une mémoire, Paris, La Découverte.

THOMAS William et ZNANIECKI Florian $(1918,1998)$ Le paysan polonais en Europe et en Amérique, Paris, Nathan.

TOURAINE Alain $(1973,1993)$ Production de la société, Seuil, Folio-Point.

VERGÈS Françoise (2004) Postcolonial Challenges, in Nicholas Gane, ed. The future of social theory, Londres, Continuum.

\section{RÉSUMÉS}

Le contexte de la France contemporaine, entre mutations de l'Etat-Nation, sortie de l'ère industrielle et réminiscences postcoloniales, constitue un espace inédit d'observation des tensions et concurrences entre groupes majoritaires et minoritaires. Ces tensions et compétitions concernent particulièrement le comportement sexué des Français d'ascendance migrante et coloniale et révèlent les conflits de loyauté et de norme qui agitent la société française. Leurs efforts d'individuation se heurtent à une conception anachronique de la conformité, illustrée par les injonctions paradoxales dont ils font l'objet et qui trouvent dans l'identité sexuée et le comportement sexuel un terrain idéal d'imposition. Les assignations à la différence entre lesquelles ils circulent ignorent la complexité et l'ambiguïté des choix qu'ils affrontent sans garantie de légitimation et contribuent à les déposséder d'eux-mêmes.

In Dubious Battle. Competition for the Gendered Conformation of French Citizens with a Migrant and Colonial Background.

Contemporary France which is characterized by mutations of the Nation-State, the exit of the industrial era and post-colonial reminiscence is an original space to observe tensions and competition between majority and minority groups. These tensions and competitions concern mainly the gendered behavior of French citizens with an immigration and colonial background and reveal conflicts of loyalty and norms in French society. The article deals with these issues by stressing the importance of gendered identities.

En una lucha dudosa. Concurrencia para la conformación sexuada de los franceses de ascendencia migrante o colonial.

El contexto de la Francia contemporánea, entre las mutaciones del estado-nación , la salida de la era industrial y las reminiscencias postcoloniales, constituye un espacio inédito de observación de las tensiones y concurrencias entre grupos mayoritarios y minoritarios. Estas tensiones y competiciones conciernen de manera particular el comportamiento sexuado de los franceses de ascendencia migrante y colonial, y revelan los conflictos de lealtad y de norma que agitan a la sociedad francesa. Sus esfuerzos de individuación se enfrentan a una concepción anacrónica de la conformidad, que ilustran las exhortaciones paradoxales dirigidas hacia ellos, y que se imponen con fuerza particular en el terreno de la identidad sexuada y del comportamiento sexual. Las exigencias de diferencia entre las cuales circulan ignoran la complejidad y la ambigüedad de las alternativas entre las cuales tienen que elegir, que afrontan sin garantía de legitimación y que contribuyen a despojarlos de ellos mismos. 
INDEX

Mots-clés : genre, relations interethniques, sexe

Index géographique : France

\section{AUTEUR}

\section{NACIRA GUÉNIF-SOUILAMAS}

Maître de conférences à l'Université Paris 13 Villetaneuse, UFR Lettres et Sciences Humaines, 99 Av. J.-B. Clément, 93430 Villetaneuse, France, naciraguenif@wanadoo.fr 